\title{
Sol-Gel Microcapsulation in Silica-Based Particles: A Comparative Study
}

ARTICLE in SILICON · APRIL 2011

Impact Factor: $1.07 \cdot$ DOI: 10.1007/s12633-011-9072-0

4 AUTHORS, INCLUDING:

\section{Giuseppe Alonzo}

Università degli Studi di Palermo 121 PUBLICATIONS 855 CITATIONS SEE PROFILE

\section{Mario Pagliaro}

Italian National Research Council 177 PUBLICATIONS 3,248 CITATIONS
Rosaria Ciriminna

Italian National Research Council 158 PUBLICATIONS $\quad 2,892$ CITATIONS

SEE PROFILE

SEE PROFILE 


\section{Sol-Gel Microcapsulation in Silica-Based Particles: A Comparative Study}

\section{Marzia Sciortino, Giuseppe Alonzo, Rosaria Ciriminna \& Mario Pagliaro}

\section{Silicon}

ISSN 1876-990X

Volume 3

Number 2

Silicon (2011) 3:77-83

DOI $10.1007 / \mathrm{s} 12633-011-9072-0$

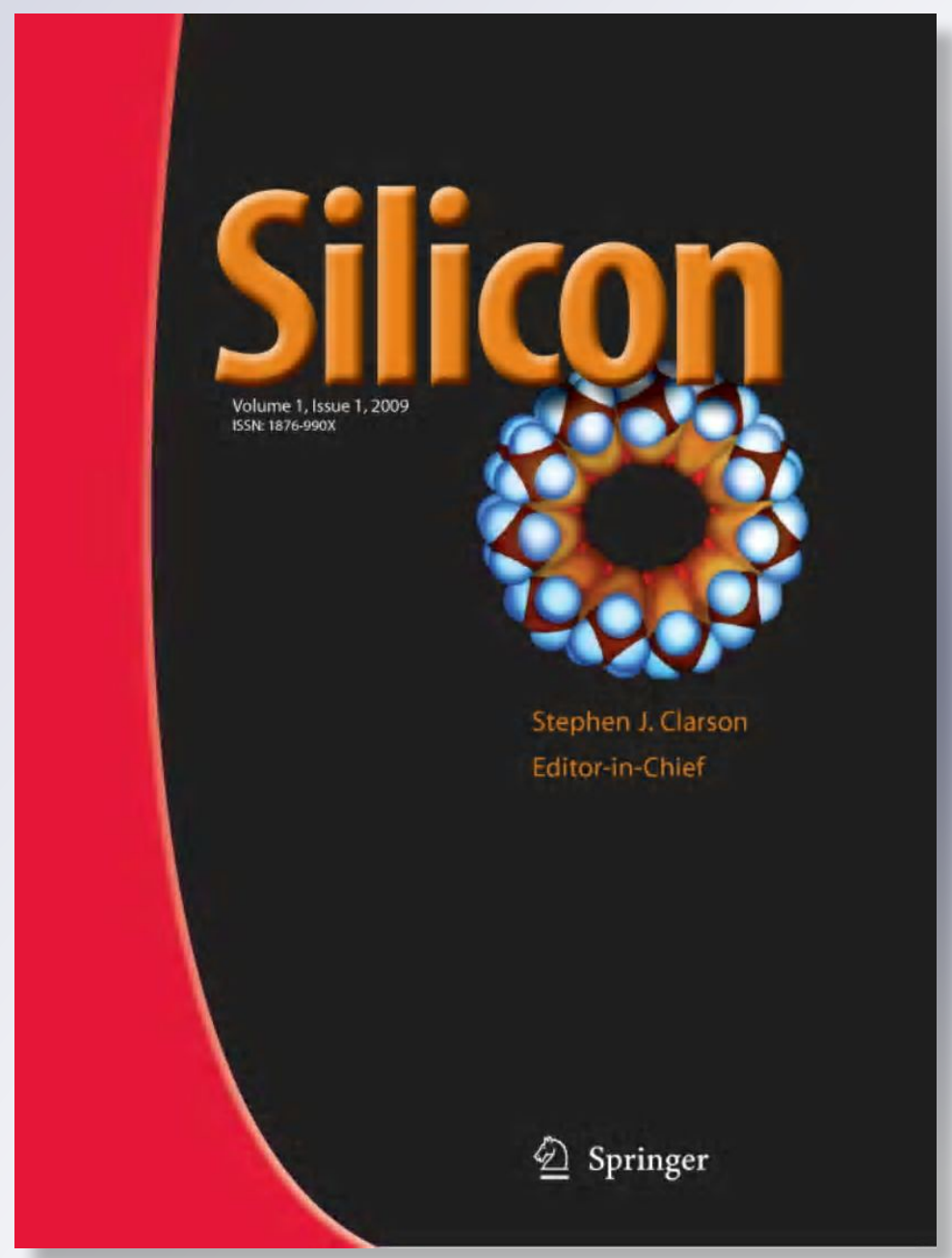

\section{照 Springer}


Your article is protected by copyright and all rights are held exclusively by Springer Science+Business Media B.V.. This e-offprint is for personal use only and shall not be selfarchived in electronic repositories. If you wish to self-archive your work, please use the accepted author's version for posting to your own website or your institution's repository. You may further deposit the accepted author's version on a funder's repository at a funder's request, provided it is not made publicly available until 12 months after publication. 


\title{
Sol-Gel Microcapsulation in Silica-Based Particles: A Comparative Study
}

\author{
Marzia Sciortino • Giuseppe Alonzo • \\ Rosaria Ciriminna $\cdot$ Mario Pagliaro
}

Received: 26 November 2010 / Accepted: 19 January 2011 / Published online: 12 February 2011

(C) Springer Science+Business Media B.V. 2011

\begin{abstract}
Comparison between the two main sol-gel/ emulsion methods to prepare microparticles made of organosilica doped with a lipophilic molecule shows that entrapment only takes place starting from $\mathrm{O} / \mathrm{W}$ emulsions. In this case, however, formation of spherical microcapsules, observed when the sol-gel polycondensation is carried out in a $\mathrm{W} / \mathrm{O}$ microemulsion, does not take place.
\end{abstract}

Keywords Microparticle · Sol-gel · Microencapsulation · Emulsion $\cdot$ Silica $\cdot$ Organosilica

\section{Introduction}

Silica and organosilica microparticles doped with functional molecules are increasingly important in a number of different applications [1] since in the research of chemical companies new molecules are still important but new effects, new systems and new functional materials are needed [2]. Basically, two main methods exist to produce silica-based microparticles doped with actives. Both use a combined microemulsion/sol-gel process. In one case the sol-gel process is combined with a water-in-oil (W/O) emulsion [3]; and in the other the sol-gel polycondensation is carried out in an oil-in-water $(\mathrm{O} / \mathrm{W})$ microemulsion [4].

\footnotetext{
M. Sciortino $\cdot$ R. Ciriminna $\cdot$ M. Pagliaro $(\triangle)$

Istituto per lo Studio dei Materiali Nanostrutturati, CNR, via U. La Malfa 153 ,

90146 Palermo, Italy

e-mail: mario.pagliaro@cnr.it

M. Sciortino $\cdot$ G. Alonzo

Dipartimento di Ingegneria e Tecnologie Agro Forestali,

Università degli Studi di Palermo,

viale delle Scienze, 13 ,

90128 Palermo, Italy
}

In this study we compare the two methods using different solvents and surfactants using in each case benzoyl peroxide (BPO) as an active dopant. In addition, we study the morphpology of the resulting particles upon varying the nature of the organosilane and the $\mathrm{pH}$ of the sol-gel process. Our aim was twofold: first, to assess how much of a dopant is incorporated and which factors influence the materials structure. Second, to study the effect of the organic modification of the silica surface. The results point to a number of relevant findings of general value.

\section{Experimental Section}

Silica and organosilica materials doped with BPO were prepared by conducting the sol-gel process in $\mathrm{W} / \mathrm{O}$ and in $\mathrm{O} / \mathrm{W}$ emulsions (Scheme 1).

Several organosilica-based particles were first prepared according to the $\mathrm{W} / \mathrm{O}$ polycondensation methodology, by changing both the organically modified alkoxide and the organic solvent (Table 1). We then prepared $\mathrm{SiO}_{2}$ and $10 \%$ alkyl-modified silica materials under either basic conditions (Table 2) or acidic conditions (Table 3).

All chemicals were purchased from Aldrich and used without further purification. A typical preparation of organosilica doped particles in a water-in-oil (W/O) emulsion was prepared by mixing $50 \mathrm{mg}$ of BPO dissolved in tetraethylorthosilicate (TEOS, $5.00 \mathrm{~mL}$ ) along with $\mathrm{MeOH}(1.00 \mathrm{~mL})$ and water $(3.50 \mathrm{~mL})(\mathrm{Si}: \mathrm{MeOH}$ : $\mathrm{H}_{2} \mathrm{O}=1: 1: 8$ molar ratio). This mixture was added with methyltrimethoxysilane (MTMS, $350 \mu \mathrm{l}$ ). The resulting solution was stirred for $24 \mathrm{~h}$ at ambient temperature. Then a surfactant solution was prepared by dissolving a surfactant (e.g. Span 85) in $50 \mathrm{~mL}$ of non-polar solvent (e.g. hexane or cyclohexane) followed by homogenization using an 


\section{Sol-gel microcapsules $(W / 0)$}
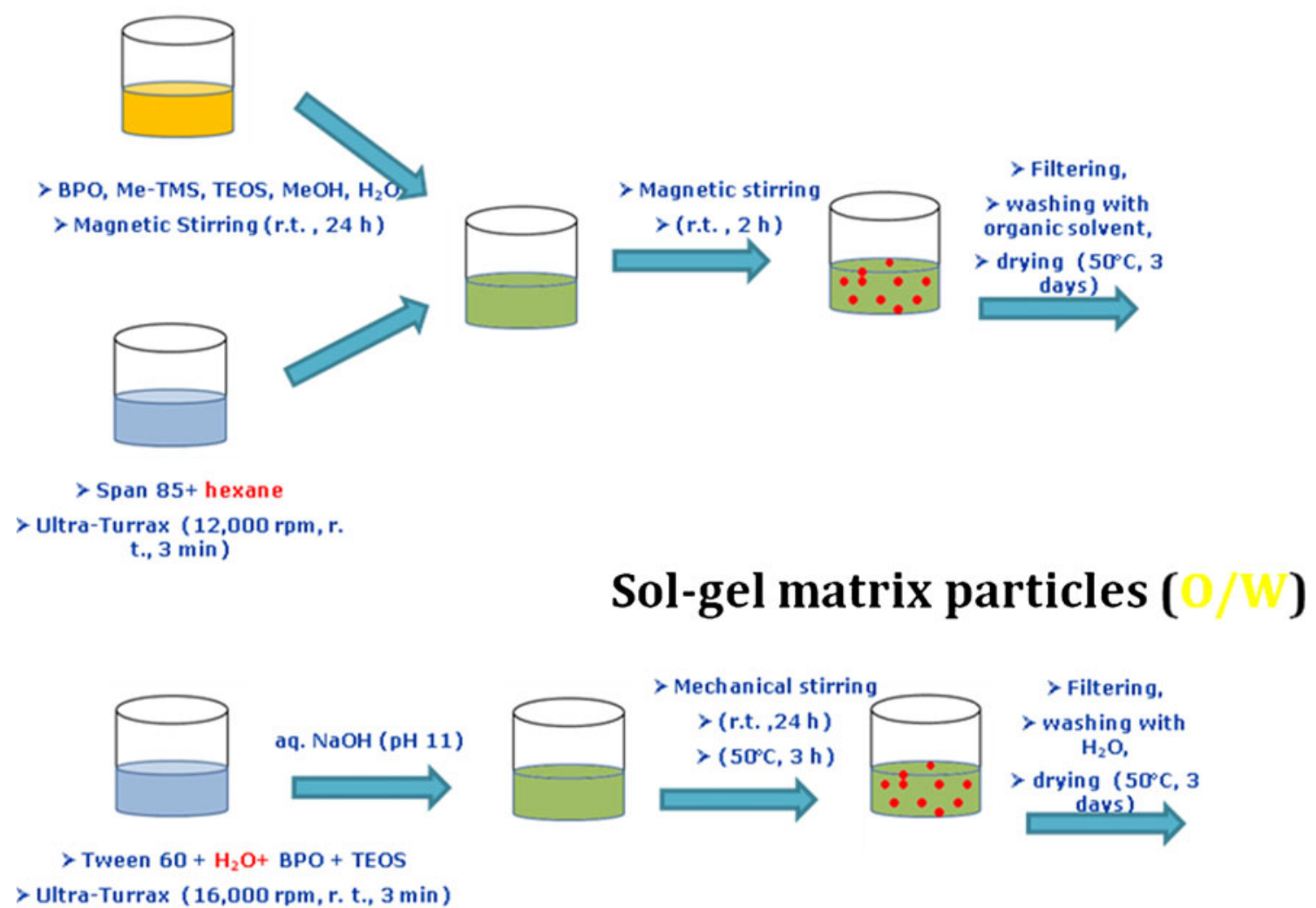

$>$ Ultra-Turrax $(16,000 \mathrm{rpm}$, r. t., $3 \mathrm{~min})$

Scheme 1 Sol-gel routes to silica-based microparticles in different emulsions

Ultra-Turrax T-25 basic with S 25 KR-18G dispersing tool (IKA) at 19,000 rpm for 2 min to form a clear solution to which was added the sol-gel solution $(800 \mu l)$. The resulting emulsion was stirred for $60 \mathrm{~min}$ at $500 \mathrm{rpm}$ resulting in the formation of a suspension of white microparticles. The filtrate was washed 4 times with the nonpolar solvent and the material dried at $50{ }^{\circ} \mathrm{C}$ for 2 days.

A typical preparation of organosilica particles in a oil-inwater emulsion $(\mathrm{O} / \mathrm{W})$ was prepared by dissolving $665 \mathrm{mg}$ of benzoyl peroxide in $16 \mathrm{~mL}$ TEOS and $1.15 \mathrm{~mL}$ MTMS. The organic phase was emulsified in $100 \mathrm{~mL}$ of aqueous solution containing the surfactant Tween $60(1 \% \mathrm{w} / \mathrm{w})$ under high shear forces using an Ultra-Turrax T-25 basic with S $25 \mathrm{KR}-18 \mathrm{G}$ dispersing tool (IKA) at 19,000 rpm. This emulsion was then poured into a flask containing $100 \mathrm{~mL}$ $\mathrm{NaOH}$ aqueous solution at $\mathrm{pH}$ 11.3. The solution was stirred at $400 \mathrm{rpm}$ while the emulsion was added, and then the rate was set at $200 \mathrm{rpm}$. The mixture was stirred at room temperature for $24 \mathrm{~h}$, followed by stirring at $50{ }^{\circ} \mathrm{C}$ for $3 \mathrm{~h}$. The resulting suspension was washed with deionized water and freeze-dried.

SEM measurements were carried out using the Hitachi 5570 Scanning Electron Microscope. TEM experiments were done with a Hitachi H7650 Transmission Electron Microscope. The optical images were obtained with a Carl Zeiss optical
Table 1 Synthetic protocol for microparticles doped with BPO from $\mathrm{W} / \mathrm{O}$ emulsions

For each sample, except BPO@TMOS/MTMS (hexane), the amounts of BPO, $\mathrm{MeOH}$, $\mathrm{H}_{2} \mathrm{O}$ and Span 85 added were, respectively, $50 \mathrm{mg}, 1 \mathrm{~mL}$, $3.5 \mathrm{~mL}$ and $9.6 \mathrm{~g}$. All preparation employed $50 \mathrm{ml}$ of oil (hexane or cyclohexane)
BPO@TMOS/MTMS (hexane)

$350 \mu 1$ MTMS

3,50 $\mathrm{ml}$ TMOS

BPO@TMOS/MTMS (cyclohexane)

$350 \mu 1 \mathrm{MTMS}$

3,50 ml TMOS

BPO@TEOS/MTMS (hexane)

$350 \mu 1 \mathrm{MTMS}$

5,00 $\mathrm{ml}$ TEOS
BPO@, TEOS/PTMS (hexane)

$440 \mu 1$ PTMS

5,00 $\mathrm{ml}$ TEOS

BPO@TEOS/PhTMS (hexane)

$470 \mu 1$ PhTMS

5,00 $\mathrm{ml}$ TEOS

3,46 g Span 20

BPO@TEOS/MTMS (hexane)

$350 \mu 1$ MTMS 350

$5,00 \mathrm{ml}$ TEOS 
microscope. DRIFT FTIR spectra were recorded with a Mattson RS1 spectrometer, with a wide range MCT detector, at $4 \mathrm{~cm}^{-1}$ resolution, resulting from 1,000 scans for the sample ratioed against the same number of scans for the background. The BET pore size and specific surface area were obtained on a Micromeritics ASAP 2050 V1.00 E sorption analyzer.

\section{Results and Discussion}

The selection of the surfactant is critical in controlling the interfacial tension between the non-polar and polar phase. In the case of W/O reactions, surfactants Span 80 (Sorbitan monooleate, 1) and Span 85 (Sorbitan trioleate, 2) were selected as emulsifying agents because their $\operatorname{HLB}^{1}$ (4.3 and 1.8 , respectively) is suitable for water-in-oil emulsion. Surfactant Tween 60 (Polyethylene glycol sorbitan monostearate, 3; HLB = 14.9) was chosen for the O/W syntheses.<smiles>CCCCCCCCCCCCC(=O)OCC(O)C1OCC(O)C1O</smiles>

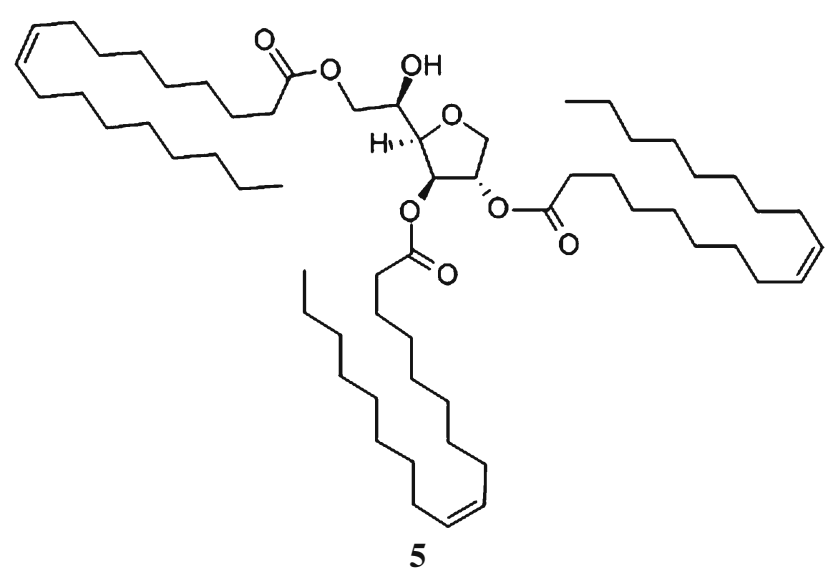

2

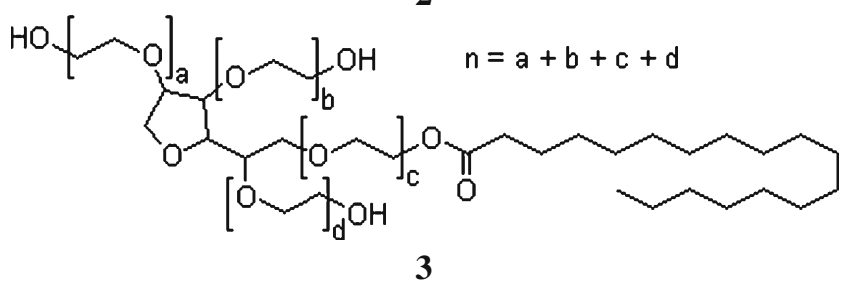

\footnotetext{
${ }^{1}$ The HLB number is used as a measure of the ratio of these groups. It is a value between 0 and 60 defining the affinity of a surfactant for water or oil. HLB numbers are calculated for nonionic surfactants, and these surfactants have numbers ranging from 0 to 20. HLB numbers $>10$ have an affinity for water (hydrophilic) and number $<10$ have an affinity of oil (lipophilic). Ionic surfactants have recently been assigned relative HLB values, allowing the range of numbers to extend to 60. See also: Myers [5].
}

Table $2 \mathrm{SiO}_{2}$ and $10 \%$ alkyl-modified silica particles prepared from $\mathrm{O} / \mathrm{W}$ emulsions at $\mathrm{pH} 11$

\begin{tabular}{cccr}
\hline Sample & TEOS $(\mathrm{mL})$ & Organosilane $(\mathrm{mL})$ & Yield $(\mathrm{g})$ \\
\hline BPO 4 & $100 \%(17,5)$ & - & 6,18 \\
BPO 5 & $90 \%(16)$ & $10 \%$ MTMS $(1,55)$ & 4,554 \\
BPO 6 & $90 \%(16)$ & $10 \%$ MTMS $(1,15)$ & 4,829 \\
BPO 7 & $90 \%(16)$ & $10 \%$ PTMS $(1,4)$ & 5,129 \\
\hline
\end{tabular}

Tween 60 is the surfactant employed. MTMS methyltrimethoxysilane; PTMS propyltrimethoxysilane

In general, the method gave nice spherical microparticles. For example, the optical microphotograph in Fig. 1 shows the particles obtained from a $10 \%$ methyl-modified silica sample obtained using hexane as organic solvent (BPO@TMOS/MTMS).

The "ghost shell" in the image is a common optical artifact with glass spheres, and does not point to a core shell structure. Indeed, the SEM picture (Fig. 2) of the same materials clearly shows the formation of full organosilica matrix particles of varying diameter, around $100 \mu \mathrm{m}$.

What is remarkable from an applicative viewpoint, however, is that no BPO was entrapped in the resulting materials pointing to complete leaching of the hydrophobic molecule into the oil phase, in which it is much more soluble, eventually affording empty microparticles. Accordingly, the FTIR spectrum of the material (Fig. 3) displays the typical vibrational modes of an organically modified silica: the broad $\mathrm{VOH}\left(\sim 3,300 \mathrm{~cm}^{-1}\right)$, the $\gamma_{\mathrm{as}} \mathrm{CH}_{3}$ and $\nu_{\mathrm{s}} \mathrm{CH}_{3}$ (at 2,964 and $2,887 \mathrm{~cm}^{-1}$, respectively), the $\delta \mathrm{Si}-\mathrm{C}$ $\left(1,254 \mathrm{~cm}^{-1}\right)$, the $\gamma_{\text {as }}$ Si-O-Si $\left(1,080 \mathrm{~cm}^{-1}\right)$, the $v \mathrm{Si}-\mathrm{O}$ $\left(950 \mathrm{~cm}^{-1}\right)$, and the $v_{\mathrm{s}} \mathrm{Si}-\mathrm{O}-\mathrm{Si}\left(800 \mathrm{~cm}^{-1}\right)$ partially overlapped with the $\rho(\mathrm{O})-\mathrm{CH}_{3}\left(830 \mathrm{~cm}^{-1}\right)$. The main bands of benzoyl peroxide would be the in-phase and out-of-phase $\checkmark \mathrm{C}=\mathrm{O}$ modes (a doublet at $1,780 / 1,760 \mathrm{~cm}^{-1}$ ), the aromatic ring $\vee \mathrm{C}-\mathrm{C}$ modes $\left(1,455 \mathrm{~cm}^{-1}\right)$ and the $\vee \mathrm{O}-\mathrm{O}\left(700 \mathrm{~cm}^{-1}\right)$, all in wavenumber ranges of very low absorption from the matrix. Since they are absent, it is evident that BPO is not present within these microcapsules.

BPO is poorly soluble in water but it soluble and stable in a mixture of water and hydric solvents such as $\mathrm{MeOH}$ [6], in which we dissolved the substance for the sol-gel encapsulation. The reason for its absence from the sol-gel

Table $3 \mathrm{SiO}_{2}$ and $10 \%$ methyl-modified silica particles prepared from $\mathrm{O} / \mathrm{W}$ emulsion at $\mathrm{pH} 2$

\begin{tabular}{lccr}
\hline Sample & TEOS $(\mathrm{mL})$ & Organosilane $(\mathrm{mL})$ & Yield $(\mathrm{g})$ \\
\hline BPO 8 & $90 \%(16)$ & $10 \%$ MTMS $(1,15)$ & 3,42 \\
BPO 9 & $100 \%(17.5)$ & - & $4,10 \mathrm{~g}$
\end{tabular}

Tween 60 is the surfactant employed 


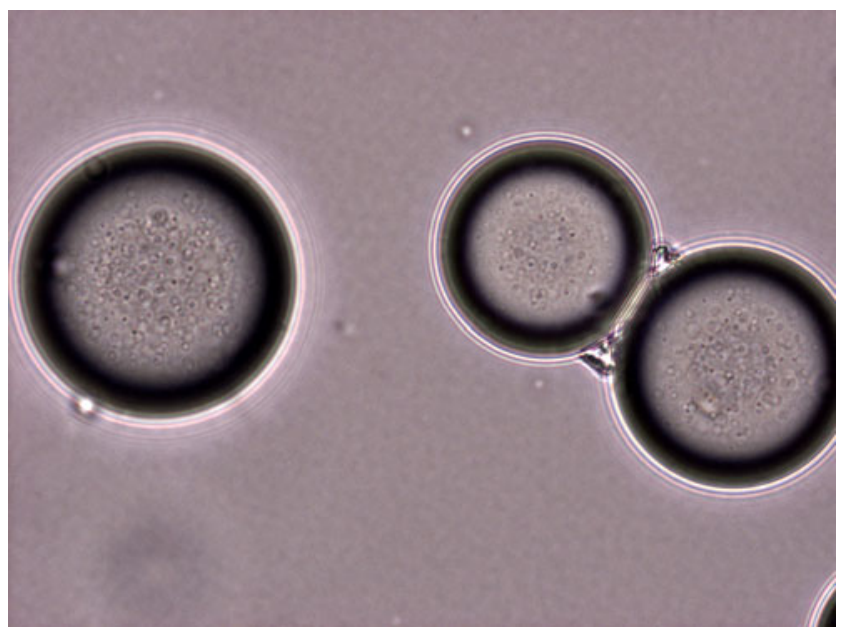

Fig. 1 Optical micrograph of BPO@TMOS/MTMS (hexane), a sol-gel 10\% methyl-modified silica matrix particles obtained using a water-in-oil emulsion with hexane as organic solvent and Span 85 as surfactant

particles is that BPO is highly lipophilic, a favorable characteristic for penetration into sebaceous follicles in the anti-acne treatment with silica microcapsules with $\mathrm{BPO}^{2}$ when all released BPO molecules migrate from the polar emulsion droplets to the follicles.

This result shows that the $\mathrm{W} / \mathrm{O}$ approach to silica microparticles is suitable exclusively for the microencapsulation of hydrophilic molecules, beyond the requirement of solubility in water or in protic solvents [7]. It other words, not only a molecule must be soluble in the water/ alcohol original sol, but it must not be liphophilic. Otherwise, no entrapment will take place.

We thus attempted microencapsulation using the oil-inwater $(\mathrm{O} / \mathrm{W})$ methodology developed by Avnir and coworkers [4]. Both acid and basic catalysis were employed. In each case, the method afforded particulate materials with high loads of entrapped BPO, which led us to encapsulate about $600 \mathrm{mg}$ in 4-6 g of final materials. ${ }^{3}$ Actual entrapment was directly proved by extraction of the entrapped BPO with ethyl acetate under reflux and further GC-MS analysis of the extract.

Micrographs in Fig. 4 show the SEM and TEM pictures of a $10 \%$ methyl-modified sample (BPO 4 in Table 2). Obviously, no microcapsule formation has taken place

\footnotetext{
$\overline{2}$ The Israeli company Sol-gel Technologies has developed in 2008 the technology for the manufacture of Cool Pearls BPO, a nonirritating acne therapeutic agent made of silica capsules doped with benzoyl peroxide that was recently commercialized in the US. See also at: www.sol-gel.com.

${ }^{3}$ The amount of entrapped BPO was assessed by dissolving each sample particles in strong base $(\mathrm{NaOH} 3 \mathrm{M})$ followed by extraction of BPO with ethyl acetate. In each sample the percentage of entrapped BPO was higher than $90 \%$ of the amount initially added.
}

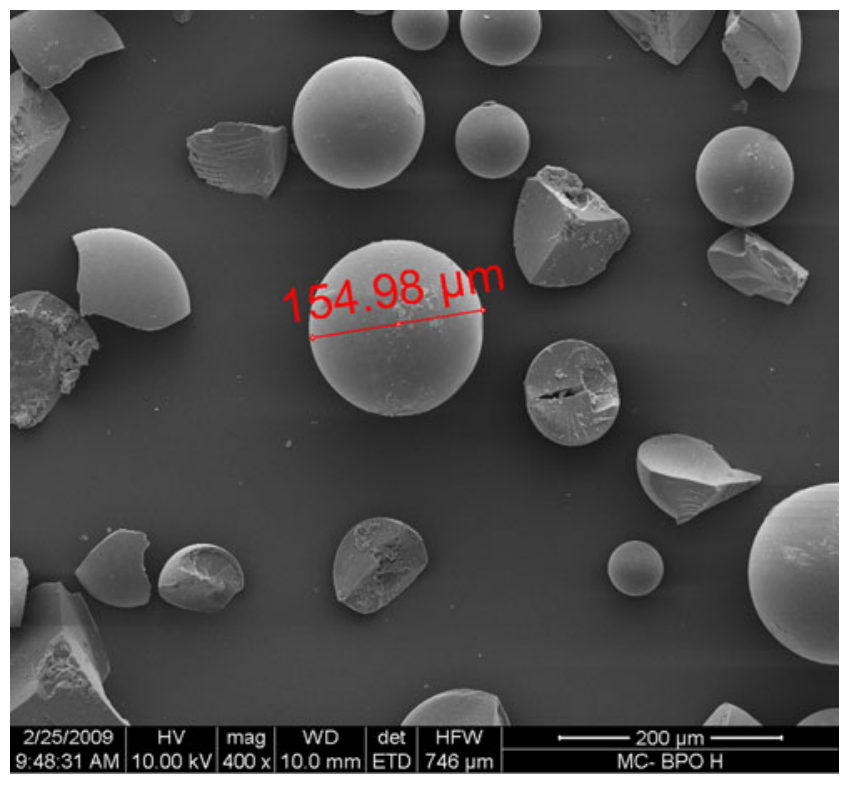

Fig. 2 SEM image of BPO@TMOS/MTMS (hexane), a sol-gel 10\% methyl-modified silica matrix particles obtained using a W/O emulsion

using the alkaline $\mathrm{O} / \mathrm{W}$ materials synthesis. Matrix particle aggregates are instead formed resembling a classical sol-gel precipitate resulting from an unstable emulsion.

We then investigated the morphology of matrix particles made of a $10 \%$ methyl-modified sample obtained under acidic conditions (BPO 8 in Table 3). This time the SEM and TEM pictures in Fig. 5 show the typical branched

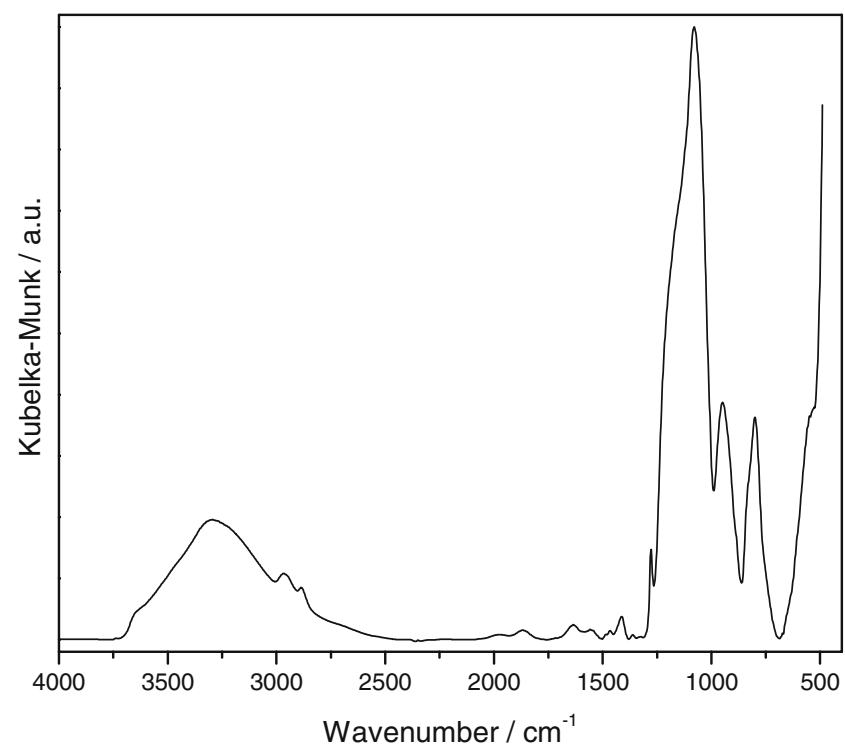

Fig. 3 DRIFT spectrum of a droplet of 10\% methyl-modified silica (BPO@TMOS/MTMS). Despite attempt to dope it with BPO, the spectrum clearly indicates that all the BPO was leached in the oil phase 
Fig. 4 SEM pictures of samples BPO 4 organosilica samples obtained under alkaline conditions. Pictures of BPO 5, BPO 6 and BPO 7 samples, not shown, are similar

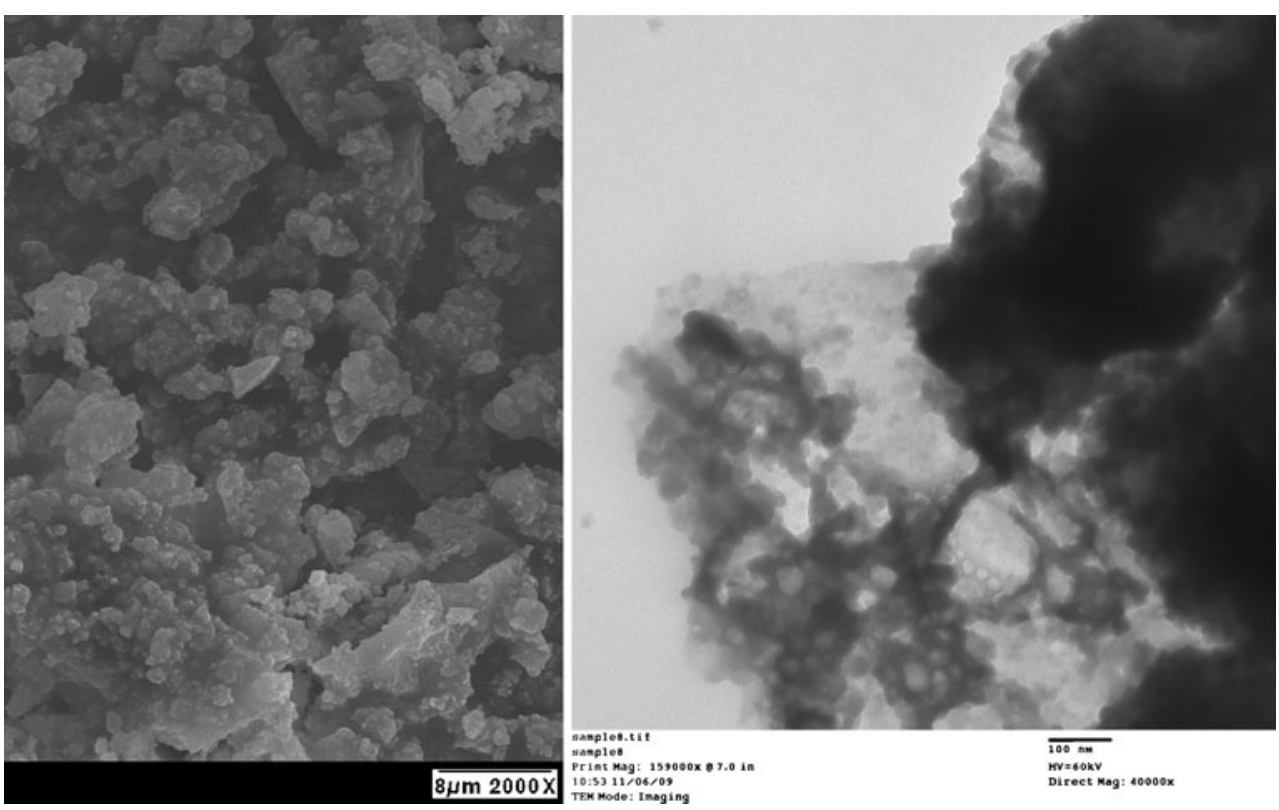

structure of sol-gels formed at acidic $\mathrm{pH}$ due to the lower condensation rate of the silane monomers and oligomers [8]. The TEM picture shows that material in this case could be made by microparticles although they are heavily aggregated/fused together (3 smaller particles, in the image at the bottom of Fig. 5).

Finally, the BET average pore diameter obtained from the BET interpolation is $92 \AA$ whereas the extremely low specific surface area $\left(0.15 \mathrm{~m}^{2} / \mathrm{g}\right)$ excludes any kind of porosity.

Based on these findings a mechanism for formation of the solid organosilica particles in the $\mathrm{O} / \mathrm{W}$ microemulsion is proposed in Scheme 2. In practice, the TEOS/ MTMS oil droplets in the water phase stabilized by the Tween 60 surfactant become unstable after addition of the basic $(\mathrm{NaOH})$ and acid $(\mathrm{HCl})$ catalysts of the sol-gel hydrolytic polycondensation. Both TEOS and the organosilane are now hydrolyzed and assembled, but the process instead than taking place at the oil-water interface, occurs at the interface of nucleated silane oligomer particles giving rise to the formation of irregular solid, matrix particles.

This process is accelerated under basic conditions, when condensation is much faster than under acidic condition, resulting in the formation of aggregates that are typical of sol-gel unstable mixtures of $\mathrm{Si}$ alkoxides. Under acidic conditions, condensation is slow and the typical branched structure of sol-gels formed at acidic $\mathrm{pH}$ occurs, resulting in compact, microporous materials of very low surface area. Supporting our hypothesis, Teng and co-workers have recently reported that in the synthesis of silica-based microparticles stabilization of the metastable emulsion phase is often required using a water/ethanol mixture in place of water only [9].

\section{Outlook and Conclusions}

In conclusion, microencapsulation of a lipophilic substance such as BPO in organosilica-based microparticles requires the employment of a $\mathrm{O} / \mathrm{W}$ emulsion to compartmentalize the active inside the oil droplets. If the $\mathrm{W} / \mathrm{O}$ approach is used, no encapsulation occurs as the BPO molecules migrate from the polar droplets to the emulsion organic phase.

Whereas in the case of the W/O emulsion, spherical microparticles of solid organosilica are obtained, no microcapsule formation occurs using $\mathrm{O} / \mathrm{W}$ sol-gel polycondensation even if high loads (ca. 10\% in weight of microparticles) could be entrapped in the particulate materials. We make the hypothesis that both acid and basic catalysts employed to catalyze the sol-gel polycondensation destabilize the metastable emulsion. The hypothesis is reinforced also by the observation of the disordered aggregate particles typical of precipitate from unstable sol-gel precursor mixtures that is due to rapid condensation of the silane monomers and oligomers under basic conditions. For industrial applications efficient microencapsulation must be accompanied by a good degree of control over the particle size. Hence, we are now studying methods to stabilize the emulsion system. The results of this report are in any case of relevance to academic and industrial researchers working for example in heterogeneous catalysis. 
Fig. 5 SEM (top) and

TEM (bottom) pictures of organosilica sample BPO

8 obtained from $\mathrm{O} / \mathrm{W}$ emulsion under acidic conditions.

Top:35× (left) and $2000 \times($ right $)$
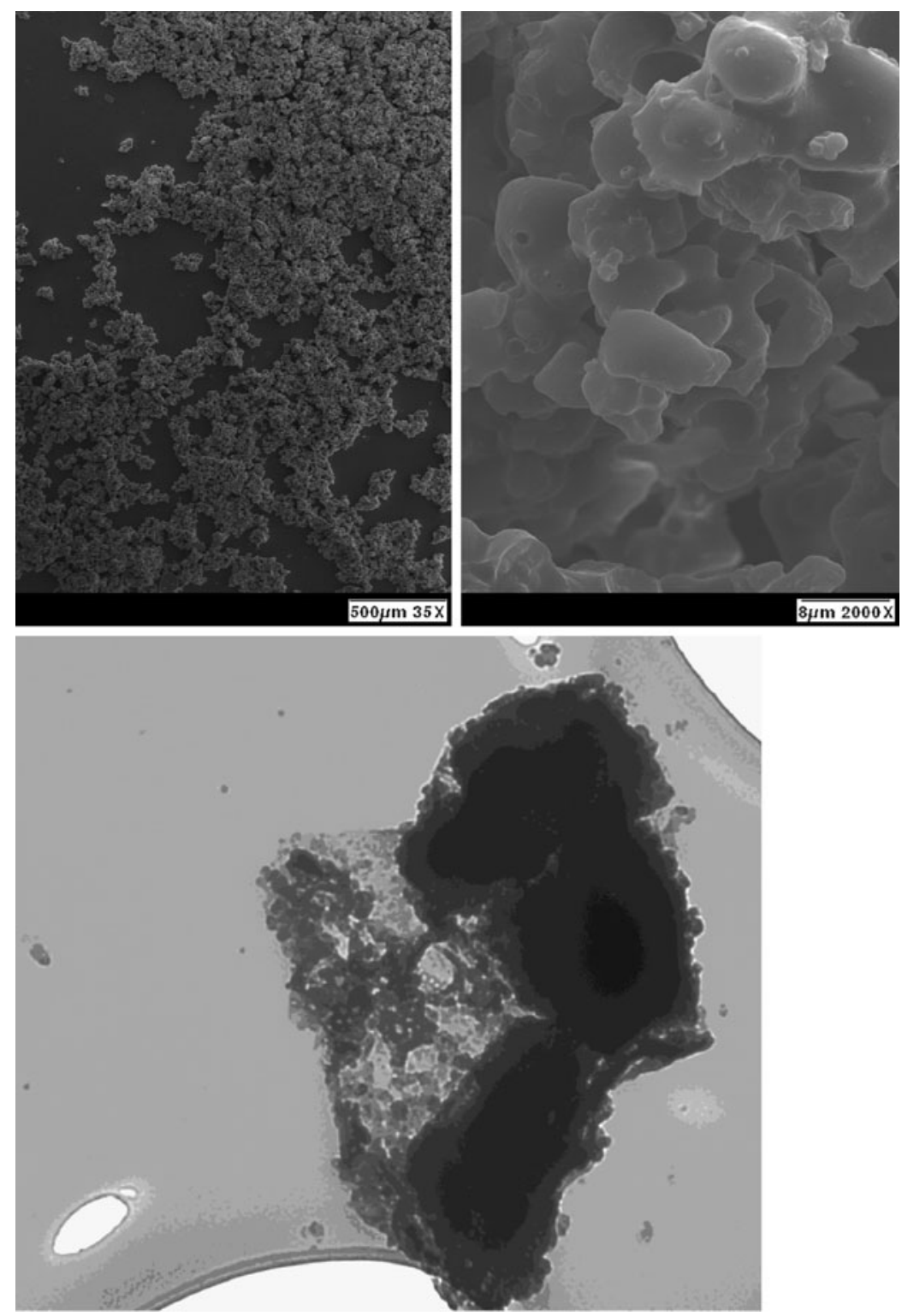

$8 \mu \mathrm{m} \mathrm{2000x}$

Scheme 2 Schematic illustration of the formation processes of solid silica matrix particles (bottom). Hollow microparticles are not formed (top) due to emulsion droplet lack of stability

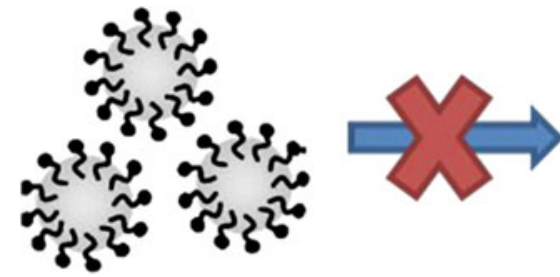

Stable TEOS/MTMS droplets
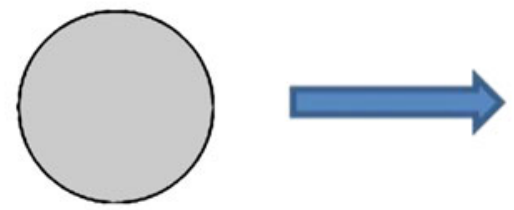

\section{Unstable TEOS/MTMS droplet}

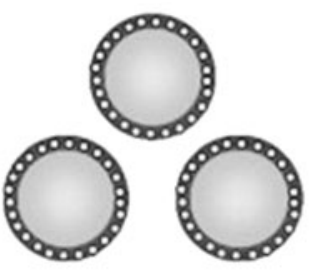

Condensation of organosilica microparticles

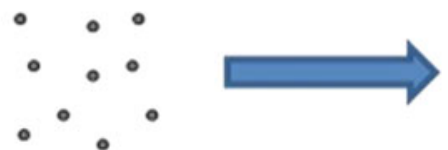

Nucleation of organosilica 
Acknowledgements This paper is dedicated to Michele and Graziella Vinci for a splendid dinner in 2004 in Milan. Thanks to Professor Bakul Dave, Southern Illinois University Carbondale, for hosting one of us (M.S.) in the context of her doctorate. We thank Professor Laura M. Ilharco, Technical University of Lisboa, for the FTIR measurements, and Dr. Vaiva Krungleviciute, Department of Physics, Southern Illinois University Carbondale, for the BET experiments.

\section{References}

1. Pagliaro M, Sciortino M, Ciriminna R, Alonzo G, De Schrijver A (2011) Chem Rev 111, xx. doi:10.1021/cr100161x
2. Kreimeyer A (BASF) (2010) BASF keeps R\&D spending at high level, press release. 28 January

3. Barbé CJA, Bartlett J, Kong L, Finnie K, Lin HQ, Larkin M, Calleja S, Bush A, Calleja G (1959) Adv Mater 2004:16

4. Lapidot N, Magdassi S, Avnir D, Rottman C, Gans O, Seri-Levy A (2003) WO 2003/80823

5. Myers D (2006) Surfactant science and technology, 3rd edn. Wiley, Hoboken, NJ

6. Chellquist EM, Gorman WG (1992) Pharm Res 9:1341

7. Barbé CJA, Kong L, Finnie KS, Calleja S, Hanna JV, Drabarek E, Cassidy DT, Blackford MG (2008) J Sol-Gel Sci Technol 46:393

8. Brinker CJ, Scherer GW (1990) Sol-gel science: the physics and chemistry of sol-gel processing. Academic Press, San Diego, CA

9. Teng Z, Han Y, Li J, Yan F, Yang W (2010) Micropor Mesopor Mater 127:67 